\title{
The Changing Epidemiology of Acute Pancreatitis Hospitalizations: A Decade of Trends and the Impact of Chronic Pancreatitis
}

\author{
Somashekar G. Krishna, MD, MPH${ }^{\star}, \dagger$, Amrit K. Kamboj, MD‡, Phil A. Hart, MD, Alice Hinton, \\ PhD§, and Darwin L. Conwell, MD, MS ${ }^{\star}$ \\ "Section of Pancreatic Disorders, Division of Gastroenterology, Hepatology, \& Nutrition, \\ Columbus, Ohio \\ †Section of Advanced Endoscopy, Division of Gastroenterology, Hepatology, \& Nutrition, \\ Columbus, Ohio \\ ₹The Ohio State University College of Medicine, Columbus, Ohio \\ $\S$ Division of Biostatistics, College of Public Heath, The Ohio State University The Ohio State \\ University Wexner Medical Center, Columbus, Ohio
}

\section{Abstract}

Objectives-The epidemiological trends contributing to increasing acute pancreatitis (AP) hospitalizations remain unknown. We sought to analyze etiological factors and outcomes of increasing AP-hospitalizations.

Methods-Utilizing the Nationwide Inpatient Sample, retrospective analyses of adult ( $\geq 18$ years) inpatient admissions with a primary diagnosis of AP (total N: 2,016,045) were performed. Patient hospitalizations from 2009-2012 were compared to those from 2002-2005.

Results-Compared to 2002-2005, there was a 13.2\% ( $\mathrm{p}<0.001$ ) increase in AP-admissions in 2009-2012. Multivariate analysis adjusted for "time-period", patient and hospital demographics, AP-etiologies, and disease associations, demonstrated an increase in the odds of associatedchronic pancreatitis (CP) (2002-2005: odds ratio [OR] 32.04, 95\% confidence interval [CI] 30.5133.64; 2009-2012: OR 35.02, 95\% CI 33.94-36.14); while associated odds of gallstones (20022005: OR 36.37, 95\% CI 35.32-37.46; 2009-2012: OR 29.85, 95\% CI 29.09-30.64) decreased.

Corresponding Author: Dr. Somashekar G. Krishna, MD, MPH, 395 W. $12^{\text {th }}$ Avenue, $2^{\text {nd }}$ floor, Division of Gastroenterology, Hepatology and Nutrition, Columbus, Ohio, USA, sgkrishna@ gmail.com, Phone: 614-293-6255.

Disclosures (Author Conflict of Interest): There are no relevant conflicts of interest to report for any author.

Author contribution:

Somashekar Krishna: study concept and design; acquisition of data; analysis and interpretation of data; drafting of the manuscript; critical revision of the manuscript for important intellectual content; study supervision

Amrit Kamboj: analysis and interpretation of data; drafting of the manuscript; critical revision of the manuscript for important intellectual content

Phil Hart: study concept and design; analysis and interpretation of data; drafting of the manuscript; critical revision of the manuscript for important intellectual content

Alice Hinton: acquisition of data; analysis and interpretation of data; drafting of the manuscript; statistical analysis; critical revision of the manuscript for important intellectual content

Darwin Conwell: study concept and design; analysis and interpretation of data; drafting of the manuscript; critical revision of the manuscript for important intellectual content 
Compared to 2002-2005, the AP-related mortality decreased in 2009-2012 (1.62\% to $0.79 \%$, $\mathrm{p}<0.001)$ and was lower in AP with associated-CP $(0.65 \%$ to $0.26 \%$; $\mathrm{p}<0.001)$ compared to AP without-CP.

Conclusion-In the preceding decade, AP-hospitalizations are increasing but associated mortality is declining. Associated-CP has emerged as a leading contributor for AP-related hospitalizations. Further research is needed to identify novel interventions to prevent disease progression of AP.

\section{Keywords}

Pancreatitis; Nationwide Inpatient Sample; epidemiology; metabolic syndrome

\section{INTRODUCTION}

Acute pancreatitis (AP) is the single most common specific gastrointestinal diagnosis for inpatient hospitalization and inpatient costs reach an estimated $\$ 2.6$ billion annually. ${ }^{1}$ The most common risk factors associated with AP are gallstones and alcohol. ${ }^{2}$. Overall, AP is associated with a significant mortality rate of $1 \%$ to $5 \%$, which has improved from historical data.

Over the last few decades, the incidence of AP in Western countries has steadily increased. ${ }^{3}$ Emergency department visits and hospitalizations for pancreatitis have also shown a steady rise paralleling the increase in incidence outlined above. ${ }^{4}$ Multiple hypotheses have been generated to explain the increased incidence of AP. While alcohol consumption may explain the trends in some countries, alcohol consumption has decreased in the US. ${ }^{5,6}$ In addition, prevalence of cigarette smoking across the US has declined between 1996 and 2012 (median $0.75 \% /$ year) ${ }^{7}$ It is presumed that gallstone-related disorders are a more probable cause of AP in the US given the rise in obesity and an increasingly aging population. ${ }^{8}$ Another major contributor for increasing detection and diagnosis of AP is more frequent laboratory testing. ${ }^{9}$ With implementation of the Affordable Care Act, the emphasis is to provide high-value care, therefor prevention of readmissions is one vital measure to reduce AP-related hospitalizations and inappropriate healthcare resource utilization.

There is a lack of large population-based studies from the US that have evaluated the role of different etiologies in explaining the increasing prevalence of AP-related hospital admissions. To further investigate this issue, we analyzed the Nationwide Inpatient Sample (NIS), the largest inpatient database in the US. Our aim was to elucidate the influence of changes in AP-related etiologies and evaluate clinically relevant hospital-based outcomes.

\section{METHODS}

\section{Data source}

The NIS Healthcare Cost Utilization Project (HCUP), an administrative claims databank, is a data compilation of more than 8 million inpatient admissions per year from approximately 1000 hospitals (representing about $85 \%$ of all nonfederal hospitals). It is designed to approximate a $20 \%$ stratified probability sample of patients from all nonfederal acute-care 
hospitals in the US. Discharge weights are provided, which allows extraction of nation-level estimates from the unweighted database information. ${ }^{10}$

The NIS-HCUP database was queried from 2002 to 2012 using the International Classification of Diseases, Ninth Revision, Clinical Modification (ICD-9-CM) diagnosis and procedure codes. Hospitalizations for AP were selected using the ICD-9-CM diagnostic code 577.1 as primary discharge diagnosis. Related etiologies and associated diagnoses were queried from secondary diagnoses using respective ICD-9-CM codes (Supplemental Table 1). The following exclusions were applied: (a) Age $\leq 17$ years; (b) Patients transferred from an outside hospital (since the duration and complications of prior hospitalization are not known); (c) All codes describing status of pregnancy.

The Ohio State University Data and Specimen Policy and Human Subjects Research Policy does not require Institutional Review Board approval for a population based public data set.

\section{Patients and outcomes}

Patient-level variables included age, sex, race, median household income for patient's zip code (quartiles), and insurance status. Race/ethnicity was categorized as white, black, hispanic, and others. Insurance status was categorized as Medicare, Medicaid, private insurance, and uninsured/other based on the primary payer listed on the discharge record. Hospital location and teaching status were combined into a single variable with the following categories: Rural, urban non-teaching, and urban-teaching hospital. Hospital bed size was classified as small, medium, or large, based on an algorithm developed by HCUP. Hospital region was classified by the U.S. Census Bureau as Northeast, Midwest, South, or West.

The following etiologies were evaluated in association with a primary diagnosis of AP: gallstone-related disorders, alcohol-related disorders, pancreatic neoplasm, current and prior history of smoking tobacco, chronic pancreatitis, and pancreatic anomalies (including pancreatic divisum). The following diseases were evaluated for associations with a primary diagnosis of AP: hyperlipidemia, obesity (including morbid obesity), cholangitis, acute cholecystitis, diabetes mellitus, non-alcoholic fatty liver disease, and metabolic syndrome. According to guidelines from the National Heart, Lung, and Blood Institute and the American Heart Association, metabolic syndrome is diagnosed when a patient has at least 3 of the following 5 conditions: Diabetes, hypertension, hypertriglyceridemia, low high density lipoprotein (HDL) cholesterol, and abdominal obesity. ${ }^{11}$ Since NIS diagnoses are based on ICD-9-CM coding (lack of specific code for low HDL cholesterol) and in an attempt to be more specific; we defined presence of metabolic syndrome when hyperlipidemia, hypertension, obesity, and diabetes were all identified in a given patientadmission.

The presence of a primary diagnosis of AP, related etiologies and disease conditions, and clinical outcomes were compared between two time-periods. A span of 4 years between 2009 and 2012 was compared to an equivalent span between 2002 and 2005. We allocated adequate time-span between the study periods to provide sufficient contrast and to prevent overlap of events. 
The primary outcome of interest was the frequency of AP-related hospital admissions and distribution of etiologies and disease associations. Additional outcomes evaluated include the effect of time-period on AP-related mortality rate, length of hospital stay, and total hospitalization costs and charges. The duration of hospital stay and incurred costs were collectively referred to as healthcare resource utilization. The amount a facility bills for a patient's care refers to the hospital charge. In contrast, hospital cost is the amount collected by a health care facility for each service and is usually less than the amount billed. To adjust for inflation, the total charges and costs were corrected to the 2012 value using the United States Bureau of Labor Statistics Consumer Price Index Inflation Calculator (http:// www.bls.gov/data/inflation_calculator.htm).

\section{Statistical Analysis}

The study schema is detailed in Figure 1. Categorical variables were tested for statistical significance with Chi-square analysis. Continuous variables were tested using the $t$ test. The mean and standard error were calculated for all continuous outcomes, and frequency counts and percentages for categorical outcomes. Statistical significance was defined by $p<0.05$. Multivariate logistic regression models were fit for each dichotomous outcome, and multivariate linear regression models were fit if the outcome variables were continuous.

Univariate analysis was performed to compare demographic characteristics, hospital features, etiologies, associated diseases, and outcomes of AP between the two time periods (2002-2005 vs. 2009-2012). Subsequently, multivariate analyses for outcomes of mortality, length of stay, and hospital costs and charges were performed. This model was adjusted for "time-period", patient and hospital demographics, etiologies, and disease associations. Results from regression models were represented using an odds ratio (OR) or coefficient, as appropriate, and $95 \%$ confidence interval (CI); all regression models were performed separately.

For analysis of predictors of changes in trends of AP-related hospital admissions, all inpatient admissions (2002-2005 and 2009-2012) for any reason were included for analysis (n: 168,474,753). A logistic regression model was fit for a primary diagnosis of AP. The model contained patient and hospital demographics in addition to variables for time-period, etiologies (gallstone-related disorders, alcohol-related disorders, pancreatic neoplasm, CP, and tobacco use), and associations (hyperlipidemia, morbid obesity, diabetes). Interactions between each etiology and/or association and "time period" were evaluated. The odds of outcome of AP for those with the time-period interaction were interpreted separately. Based on the results of the multivariate trend analysis, separate subgroup analyses were performed for the outcome of interest (presence of $\mathrm{CP}$ ) to identify predictors of AP with associated CP. Temporal trends were assessed using the Cochrane-Armitage trend test; all AP admissions from 2002 to 2012 were included.

These analyses were performed on weighted data from the NIS database using SAS 9.3 (SAS Institute, Cary, NC) employing appropriate survey procedures to produce national estimates. Aside from race (18.6\%) and income (2.5\%), there were minimal missing data for all study subjects (Supplemental Table 2). Multiple states do not document race in the discharge information, so this observation is expected. The large population-database 
facilitated exclusion of these missing data and multiple imputation was not performed since missing data were assumed to be random.

\section{Results}

\section{Temporal trends in the prevalence of AP-related admissions}

There was an increasing trend $(\mathrm{p}<0.001)$ for AP-related hospital admissions from 2002 to 2012 (Figure 2a). When compared to $2002-2005$ ( $\mathrm{n}=945,253)$, a 13.3\% ( $<$ < 0.001 ) increase in AP-related admissions was observed in $2009-2012(\mathrm{n}=1,070,792)$.

Univariate comparison of demographic and hospital factors between the two time-periods (Table 1) demonstrated that patients were younger, frequently men, belonging to lower income groups, and more frequently admitted to urban teaching hospitals. Comparison of AP-related etiologies and associated diseases between the time periods revealed that there was a marginal, albeit significant, decrease in prevalence of gallstone-related disorders. In contrast, there was an increase in prevalence of all the other etiologies and associated diseases. Notably, the greatest change was a more than $100 \%$ increase in prevalence of tobacco use and a $96 \%$ increase in prevalence of CP.

\section{Analysis of AP-related outcomes}

A 30\% decrease in AP-related mortality was noted during the more recent time period. Both unadjusted (OR $0.62,95 \%$ CI $0.58,0.65, \mathrm{p}<0.001)$ and adjusted mortality rates (OR 0.77 , $95 \%$ CI $0.72,0.83, \mathrm{p}<0.001$ ) were lower in $2009-2012$ when compared to $2002-2005$

(Table 2). Multivariate analysis also revealed a significant decrease in the length of hospital stay $(-0.78$ days, $95 \% \mathrm{CI}-0.85,-0.70 ; \mathrm{p}<0.001)$ (Table 2$)$. After controlling for inflation and adjusting for covariates, an increase in total hospital charges $(\$ 5,109,95 \%$ CI $\$ 3,976$, 6,243; $\mathrm{p}<0.001)$ was observed. However, this did not translate to an increase in hospital costs. Adjusted analysis revealed a decrease in hospital costs by $\$ 573$ per admission (95\% CI \$ -869, -277; $\mathrm{p}<0.001)$. Other variables influencing above outcomes of are listed in Supplemental Tables 3, 4, and 5.

\section{Multivariate analysis of prevalence trends of AP-hospitalizations}

A multivariate analysis model was constructed to analyze significant changes in trends of AP-related hospital admissions where the outcome was a primary discharge diagnosis of AP (Supplemental Table 6). This revealed significant interactions between most AP-related conditions and time period, with the exception of pancreatic neoplasms. Comparison of the odds of primary diagnosis of AP between the two time-periods after adjusting for demographics, hospital factors, and AP-associated etiologies and conditions is shown in table 3. Although gallstone-related disorders contributed to the highest odds of AP in the former time-period (OR 36.37, 95\% CI 35.32, 37.46), the odds were decreased during the later time-period (OR 29.85, 95\% 29.09, 30.64). Meanwhile, the odds of CP have increased and it has emerged as the condition with the highest odds of being associated with AP during the recent time period (OR 35.02, 95\% 33.94, 36.14). Although associated with increasing trends of AP, there were modest decreases in the comparative odds of alcoholrelated disorders, hyperlipidemia, and diabetes, while a minimal increase in associated 
tobacco use was observed. The presence of pancreatic neoplasm (OR 1.46; 95\% CI 1.33, 1.60) was not influenced by changing time-periods, but was associated with an increase in prevalence of AP.

\section{Acute pancreatitis with associated chronic pancreatitis}

Since CP was associated with the highest odds for a patient to be admitted with AP, we performed a subgroup analysis of this cohort to identify disease associations. There were a total of 246,577 patients discharged with a secondary diagnosis of CP. The prevalence rates of AP with associated CP more than doubled from 2002 to 2012 (18,234 [0.07\%] to 43,095 [0.16\%], $\mathrm{p}<0.001$ ) (figure 2a) while a marginal increase of prevalence rates was observed for AP-patients without CP $(0.82 \%$ [213,394] to $0.85 \%$ [226,670], $\mathrm{p}<0.001)$. Multivariate analysis (Table 4) for an outcome of AP with associated $\mathrm{CP}$ revealed that these patients were mostly men, belonging to African American race, lower socioeconomic groups, with Medicaid or Medicare coverage, and were mostly admitted to large volume, teaching urban hospitals. Associated smoking, alcohol-related disorders, and pancreatic anomalies (predominantly pancreatic divisum) had the highest odds for predicting AP with associated CP.

\section{Etiology associated mortality rates in acute pancreatitis}

For all patients with a primary diagnosis of AP, all-cause inpatient mortality decreased $(3,749[1.62 \%]$ to $2,130[0.79 \%], \mathrm{p}<0.001)$ (figure $2 \mathrm{~b}$ ). Notably this trend was parallel to the decreased mortality seen in AP with associated CP (compared to those with AP but no underlying CP) $(3,631[1.70 \%]$ to 2,020 [0.89\%], $\mathrm{p}<0.001)$. However, analysis of APpatients with a secondary diagnosis of $\mathrm{CP}$, although decreasing (118 [0.65\%] to 110 $[0.26 \%], \mathrm{p}<0.001)$, had a significantly lower mortality rate $(\mathrm{p}<0.001)$ for all the years from 2002 to 2012 .

\section{Discussion}

The prevalence of hospitalizations for AP has increased significantly in the US from 2002 to 2012. Gallstone-related disorders are a frequently associated etiology of AP, but with declining frequency. Despite an increasing prevalence of constituents of metabolic syndrome, these have modest influences on the increasing trend of AP-related hospitalizations. Rather, the increase in AP-related hospitalizations is largely due to concomitant increase in the prevalence of associated CP. Despite increasing AP-related hospital admissions, AP-associated mortality has further decreased over the last decade. Comparatively, the mortality rate in AP with associated $\mathrm{CP}$ is lower. These trends are important to identify opportunities to prevent hospitalizations related to AP and decrease avoidable utilization of healthcare resources.

Our analysis demonstrates a 16.4\% increase in AP-admissions from 2002 to 2012. Prior population-based studies have evidenced a similar increase in emergency department (ED) visits and hospital admissions for AP. ${ }^{4,12}$ While the number of hospital admissions for AP increased from 101,000 in 1988 to a peak of 210,000 in $2002,{ }^{13}$ the most recent update on the burden of gastrointestinal diseases in the US reveals a 12\% (2006 to 2012; 330,561 
visits) increase in ED visits and 15\% increase in hospital admissions (2003 - 2012; 330,561 admissions) due to AP. ${ }^{14}$ It is conceivable that the gamut of AP-admissions is comprised of many readmissions especially given the natural history of specific subsets of AP-patients. ${ }^{15}$ A population-based study of over 7,000 unique hospitalizations with AP found that over $20 \%$ were readmitted with another episode of AP. ${ }^{16}$

The conventional belief is that increasing AP is related to the global epidemic of obesity, which in turn promotes gallstone formation and can result in biliary AP. ${ }^{8,17}$ In support of obesity-related increase in new diagnosis of AP, our analysis revealed a more than 3-fold increase in associated prevalence of components of metabolic syndrome and morbid obesity. While this may explain the escalation in index cases of AP, we found that increasing APhospitalizations was most influenced by a near doubling of AP with associated $\mathrm{CP}$, and was not sufficiently supported by the rising prevalence of metabolic syndrome.

More importantly, the subgroup analysis of AP with associated CP identified a specific patient population. These patients had higher odds of tobacco use, alcohol-related disorders, African-American race, lower socioeconomic background, and were mostly covered by Medicare/Medicaid. Additionally, these patients sought care at large, urban teaching hospitals. Fortifying our findings, state-wide data (from Maryland) have also demonstrated lower mortality rates and fewer complications such as multisystem organ failure, mechanical ventilation use, or intensive care unit management in this subgroup compared to AP patients without CP. ${ }^{15}$ We also discovered an additional subset of patients with pancreatic anomalies (mostly pancreatic divisum), who had the highest odds of associated CP.

Although this study exhibited an increase in AP hospitalizations during the ten-year study period, there was a significant reduction in AP-related mortality rate and length of hospital stay. Other studies have also shown decreasing mortality rates over the last decade, which are further corroborated by our finding of a decrease from $3,749(1.62 \%)$ to $2,130(0.79 \%)$ during the study period. ${ }^{12,13,18-20}$. These outcomes are justifiable since milder cases of AP are becoming easier to detect with better testing and complications can be identified earlier in the disease course. Furthermore, recurrences after first attack of AP tend to be milder in intensity with substantially lower mortality. ${ }^{8}$

While mortality is low, the disease-related morbidity and costs of AP with associated CP is substantial. Although accounting for only 14,195 discharges in 2010, CP resulted in a cost of $\$ 150$ million, mostly due to readmissions for acute exacerbations of AP. ${ }^{14}$ With an increasing population of AP with associated $\mathrm{CP}$, these costs are expected to increase. Conceptually, although AP may progress to $\mathrm{CP}$, these are distinct clinical entities with distinct histopathology and associated clinical outcomes. ${ }^{15,21}$ It is widely acknowledged that recurrent bouts of AP increase the likelihood of developing CP and in a study that included only patients with a sentinel event of AP, specifically male gender, alcohol use, and severity of disease were predictive of readmission. ${ }^{22}$ Thus, a concerted effort is needed prevent progression of AP to $\mathrm{CP}$, which should include risk factor modification (treatment of tobacco- and alcohol-use disorders), and identification of novel therapeutic agents that prevent disease recurrence and progression. 
There are several limitations to this study. First, as with all administrative databases, coding errors represent a potential limitation of the present study. However, previous investigations evaluating the validity of codes for AP and CP suggest they are fairly accurate. A systematic review from Europe showed an accuracy of $80.3 \%$ (median) for ICD-9-CM based diagnostic coding for AP. ${ }^{23}$ A study involving 3,000 patients with a primary diagnosis of $\mathrm{CP}$ at single tertiary care hospital over 10 years (1998-2008) demonstrated a coding accuracy of $75 \% .^{24}$ Another European study observed a minimal underestimation of the discharge coding for AP and $\mathrm{CP}$ by $15.8 \%$ and $6 \%$, respectively. ${ }^{25}$ Similar to these studies, a more recent evaluation of the ICD-9-CM diagnostic coding for AP in the US reported a specificity of $85 \%{ }^{26}$ Second, the patient's race is not systematically reported by multiple states, so a definitive conclusion about the influence of race is not possible. Third, the NIS database cannot account for unobserved characteristics that may influence an intervention, complication, or outcome, so inferring 'causality' from observed associations is not valid.

Although a global improvement in healthcare has most likely decreased AP-related mortality for all etiologies, the frequency of AP related hospitalizations is increasing and $\mathrm{CP}$ represents the fastest growing associated health condition. AP with associated CP presents some distinct features, including specific subset of patients and relatively lower mortality rates while contributing to increasing health care resources. In contrast, AP not associated with $\mathrm{CP}$ demonstrates rather stable rates of hospitalization, despite the increasing prevalence of obesity and other components of metabolic syndrome. Thus, continued research addressing lifestyle factors and prevention of disease progression to $\mathrm{CP}$ are needed to further reduce the overall burden from AP-related hospitalizations.

\section{Supplementary Material}

Refer to Web version on PubMed Central for supplementary material.

\section{Acknowledgments}

Funding sources:

This publication was supported in part (PH and DC) by the National Institutes of Diabetes and Digestive and Kidney Diseases (NIDDK) and National Cancer Institute (NCI) under Award Number U01DK108327.

\section{References}

1. Peery AF, Dellon ES, Lund J, et al. Burden of gastrointestinal disease in the United States: 2012 update. Gastroenterology. 2012; 143:1179-1187. e1171-1173. [PubMed: 22885331]

2. Whitcomb DC. Clinical practice. Acute pancreatitis. N Engl J Med. 2006; 354:2142-2150. [PubMed: 16707751]

3. Spanier BW, Dijkgraaf MG, Bruno MJ. Epidemiology, aetiology and outcome of acute and chronic pancreatitis: An update. Best Pract Res Clin Gastroenterol. 2008; 22:45-63. [PubMed: 18206812]

4. Fagenholz PJ, Fernandez-del Castillo C, Harris NS, et al. National study of United States emergency department visits for acute pancreatitis, 1993-2003. BMC Emerg Med. 2007; 7:1. [PubMed: 17241461]

5. O'Farrell A, Allwright S, Toomey D, et al. Hospital admission for acute pancreatitis in the Irish population, 1997 2004: could the increase be due to an increase in alcohol-related pancreatitis? J Public Health (Oxf). 2007; 29:398-404. [PubMed: 17998260] 
6. Lowenfels AB, Sullivan T, Fiorianti J, et al. The epidemiology and impact of pancreatic diseases in the United States. Curr Gastroenterol Rep. 2005; 7:90-95. [PubMed: 15802095]

7. Dwyer-Lindgren L, Mokdad AH, Srebotnjak T, et al. Cigarette smoking prevalence in US counties: 1996-2012. Popul Health Metr. 2014; 12:5. [PubMed: 24661401]

8. Yadav D, Lowenfels AB. Trends in the epidemiology of the first attack of acute pancreatitis: a systematic review. Pancreas. 2006; 33:323-330. [PubMed: 17079934]

9. Yadav D, Ng B, Saul M, et al. Relationship of serum pancreatic enzyme testing trends with the diagnosis of acute pancreatitis. Pancreas. 2011; 40:383-389. [PubMed: 21283039]

10. (NIS) HNIS. Healthcare Cost and Utilization Project (HCUP). Agency for Healthcare Research and Quality; Rockville, MD: 2007, 2008, 2009, 2010, 2011.

11. Alberti KG, Eckel RH, Grundy SM, et al. Harmonizing the metabolic syndrome: a joint interim statement of the International Diabetes Federation Task Force on Epidemiology and Prevention; National Heart, Lung, and Blood Institute; American Heart Association; World Heart Federation; International Atherosclerosis Society; and International Association for the Study of Obesity. Circulation. 2009; 120:1640-1645. [PubMed: 19805654]

12. McNabb-Baltar J, Ravi P, Isabwe GA, et al. A population-based assessment of the burden of acute pancreatitis in the United States. Pancreas. 2014; 43:687-691. [PubMed: 24694835]

13. Fagenholz PJ, Castillo CF, Harris NS, et al. Increasing United States hospital admissions for acute pancreatitis, 1988-2003. Ann Epidemiol. 2007; 17:491-497. [PubMed: 17448682]

14. Peery AF, Crockett SD, Barritt AS, et al. Burden of Gastrointestinal, Liver, and Pancreatic Diseases in the United States. Gastroenterology. 2015

15. Akshintala VS, Hutfless SM, Yadav D, et al. A population-based study of severity in patients with acute on chronic pancreatitis. Pancreas. 2013; 42:1245-1250. [PubMed: 24152950]

16. Yadav D, O'Connell M, Papachristou GI. Natural history following the first attack of acute pancreatitis. Am J Gastroenterol. 2012; 107:1096-1103. [PubMed: 22613906]

17. Ogden CL, Carroll MD, Curtin LR, et al. Prevalence of overweight and obesity in the United States, 1999-2004. JAMA. 2006; 295:1549-1555. [PubMed: 16595758]

18. Singla A, Simons J, Li Y, et al. Admission volume determines outcome for patients with acute pancreatitis. Gastroenterology. 2009; 137:1995-2001. [PubMed: 19733570]

19. Brown A, Young B, Morton J, et al. Are health related outcomes in acute pancreatitis improving? An analysis of national trends in the U.S. from 1997 to 2003. JOP. 2008; 9:408-414. [PubMed: 18648131]

20. Krishna SG, Hinton A, Oza V, et al. Morbid Obesity Is Associated With Adverse Clinical Outcomes in Acute Pancreatitis: A Propensity-Matched Study. Am J Gastroenterol. 2015; 110:1608-1619. [PubMed: 26482857]

21. Dimcevski G, Staahl C, Andersen SD, et al. Assessment of experimental pain from skin, muscle, and esophagus in patients with chronic pancreatitis. Pancreas. 2007; 35:22-29. [PubMed: 17575541]

22. Vipperla K, Papachristou GI, Easler J, et al. Risk of and factors associated with readmission after a sentinel attack of acute pancreatitis. Clin Gastroenterol Hepatol. 2014; 12:1911-1919. [PubMed: 24815327]

23. Burns EM, Rigby E, Mamidanna R, et al. Systematic review of discharge coding accuracy. J Public Health (Oxf). 2012; 34:138-148. [PubMed: 21795302]

24. Tignor AS, Wu BU, Whitlock TL, et al. High prevalence of low-trauma fracture in chronic pancreatitis. Am J Gastroenterol. 2010; 105:2680-2686. [PubMed: 20736937]

25. Spanier BW, Schreuder D, Dijkgraaf MG, et al. Source validation of pancreatitis-related hospital discharge diagnoses notified to a national registry in the Netherlands. Pancreatology. 2008; 8:498503. [PubMed: 18765954]

26. Saligram S, Lo D, Saul M, et al. Analyses of hospital administrative data that use diagnosis codes overestimate the cases of acute pancreatitis. Clin Gastroenterol Hepatol. 2012; 10:805-811. e801. [PubMed: 22504004] 


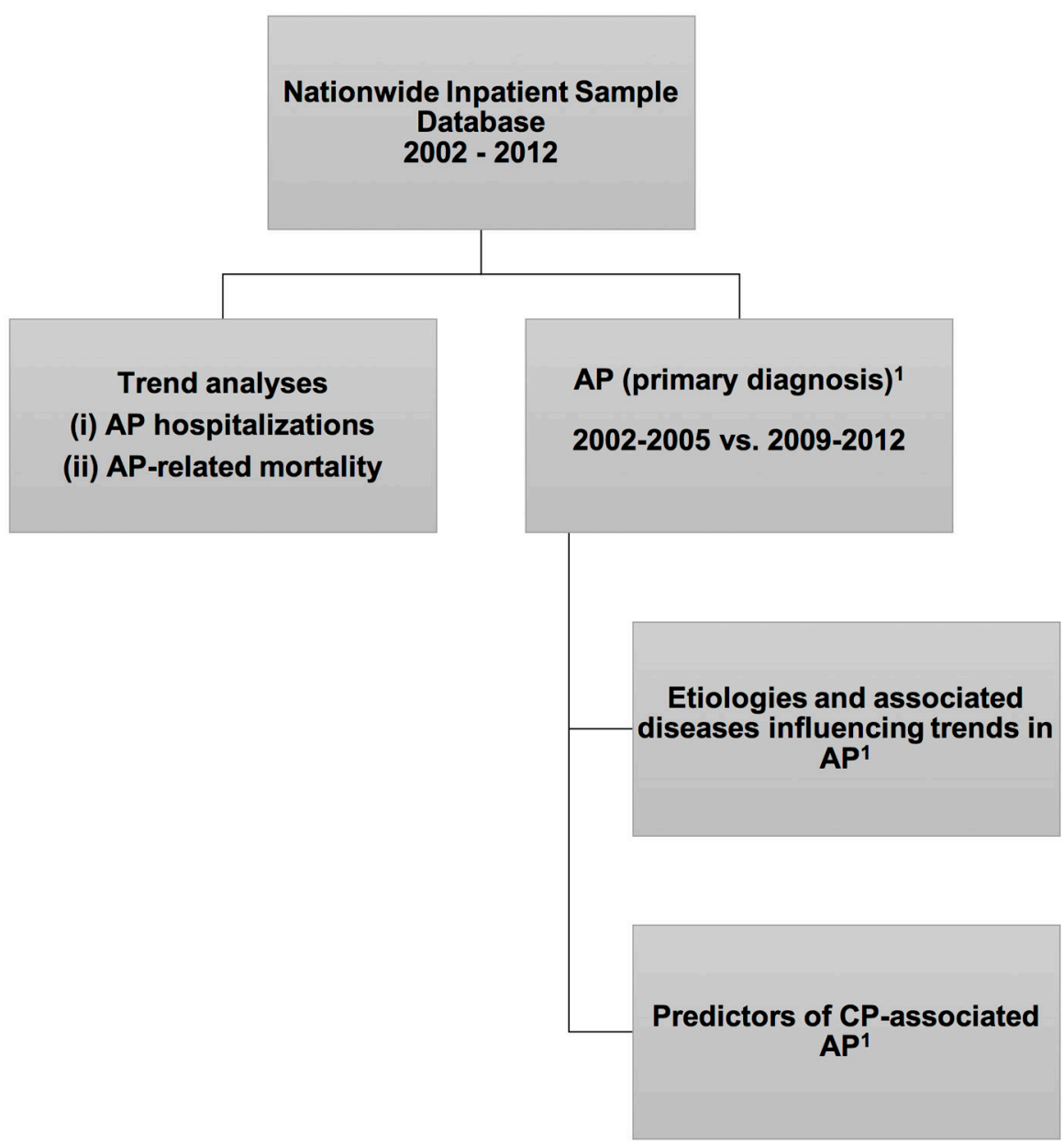

Figure 1.

Epidemiology of Acute Pancreatitis: Study schema

Foot note: AP: Acute pancreatitis; CP: Chronic pancreatitis; ${ }^{1}$ Separate multivariate analyses were performed at each step. 


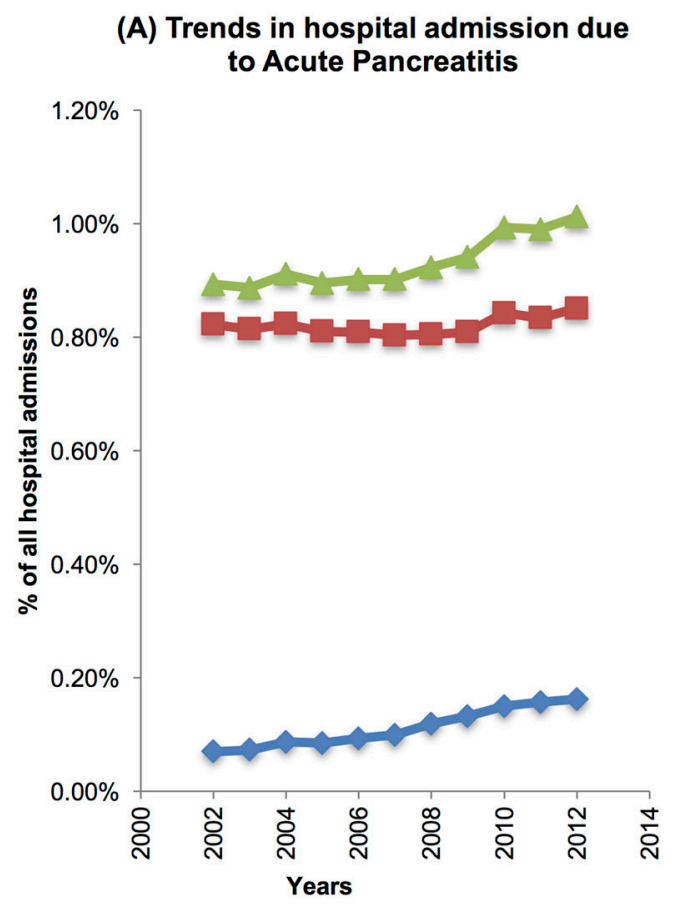

(B) Trends in mortality due to Acute Pancreatitis

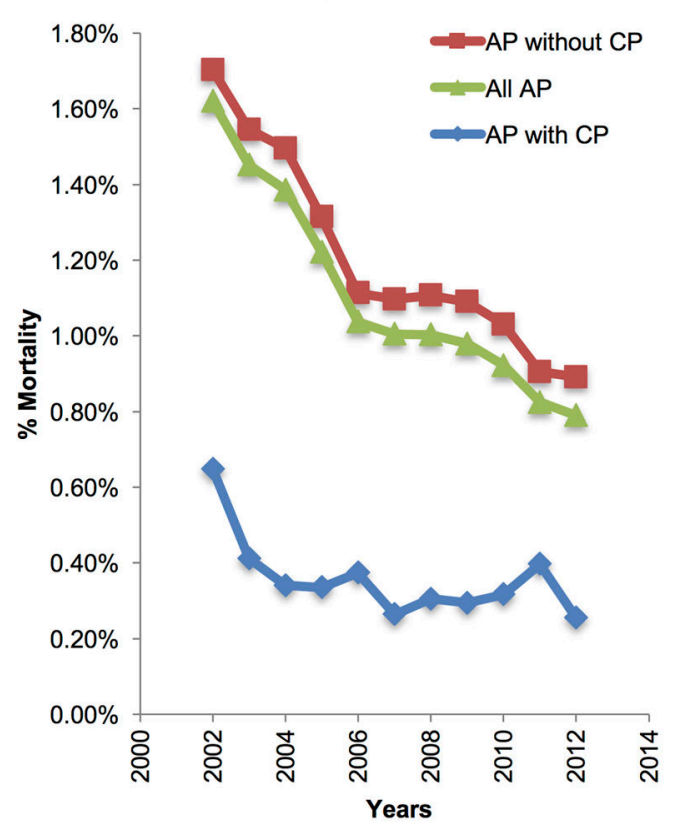

Figure 2.

Trends in inpatient hospitalization and mortality for acute pancreatitis (AP): Nationwide inpatient sample 2002 to 2012.

Foot note: CP: Chronic pancreatitis 
Krishna et al.

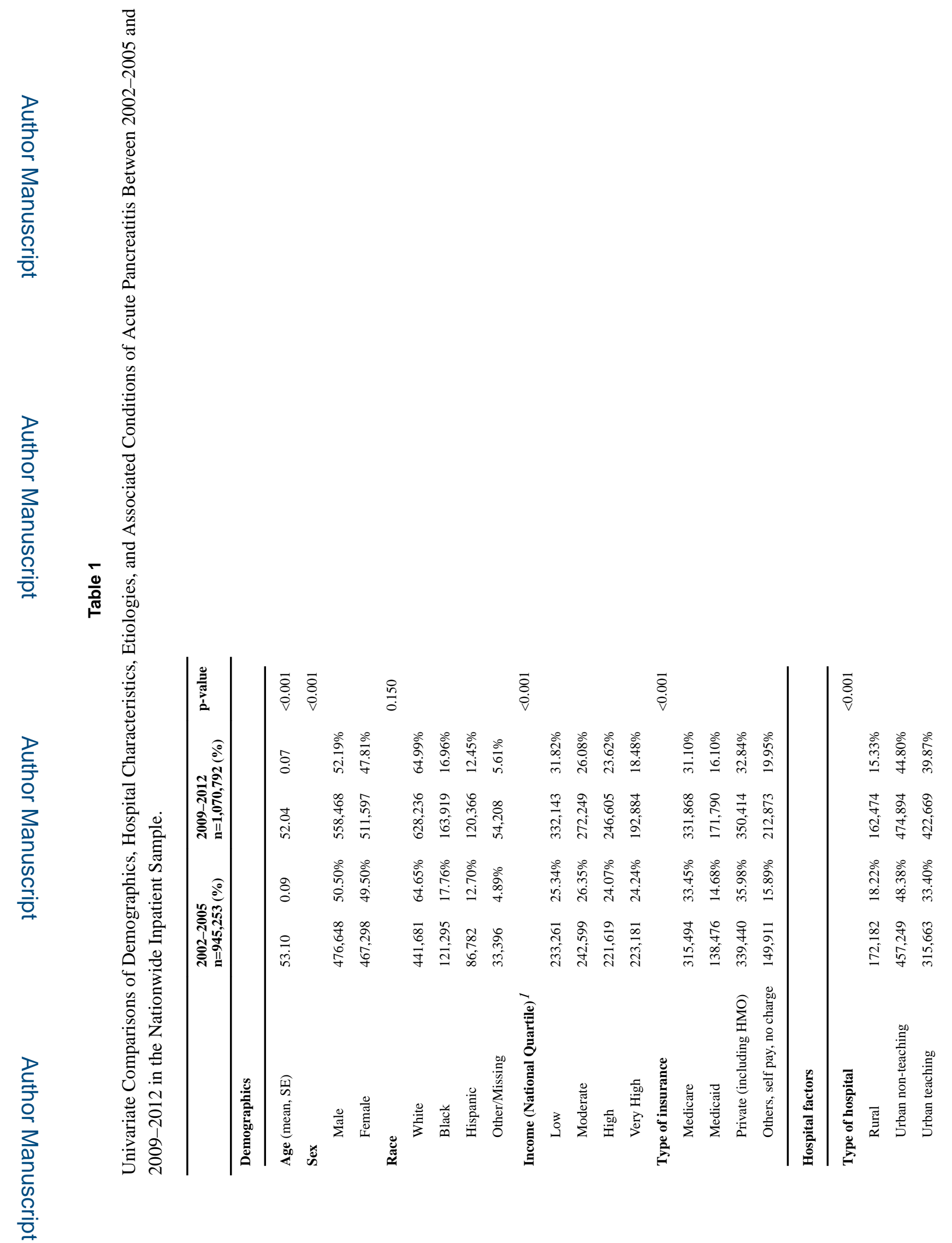

Pancreas. Author manuscript; available in PMC 2018 April 01. 
Krishna et al.

Page 13

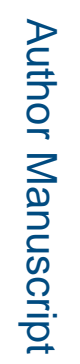

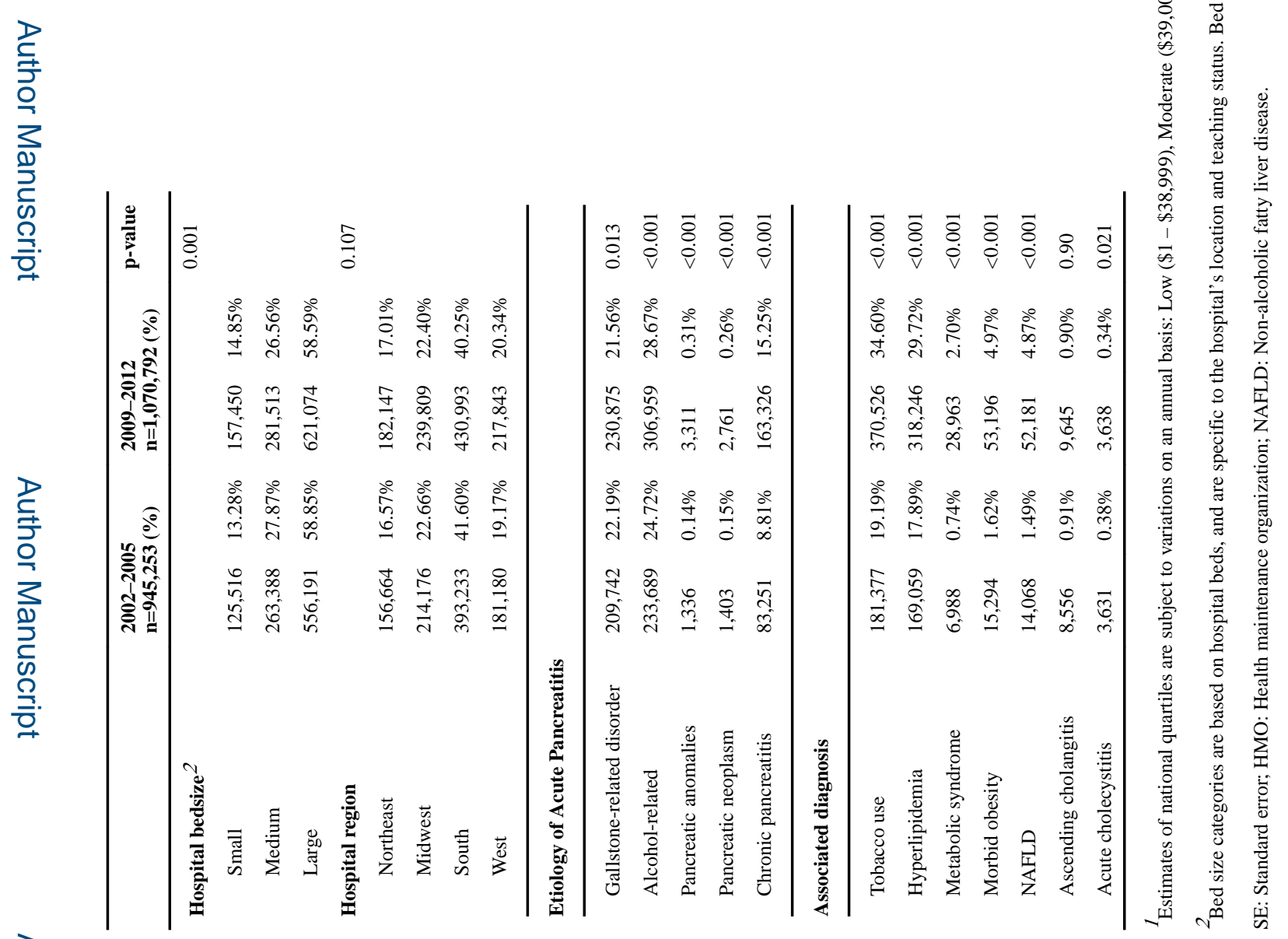

롤

Pancreas. Author manuscript; available in PMC 2018 April 01. 


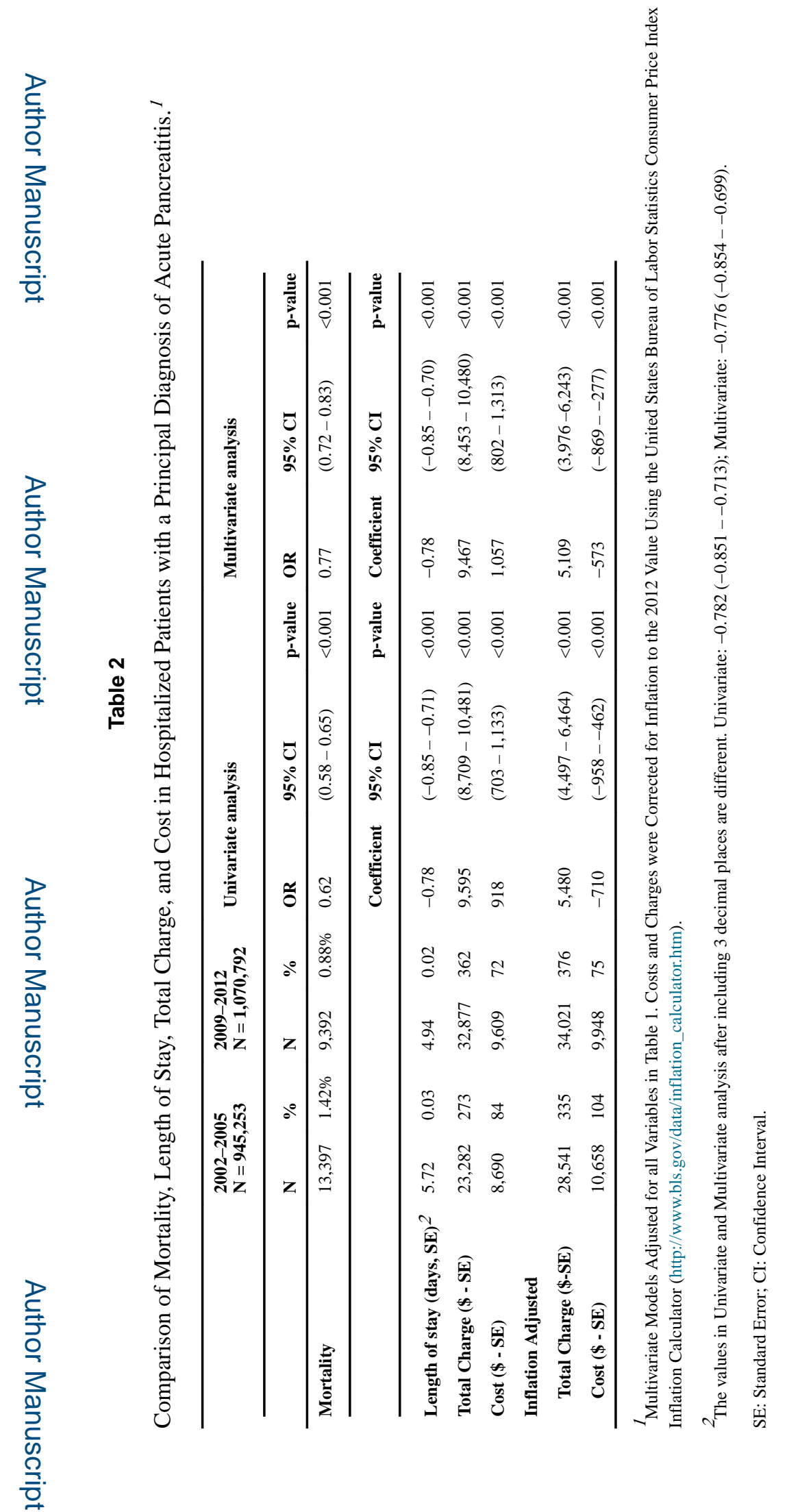

Pancreas. Author manuscript; available in PMC 2018 April 01. 


\section{Table 3}

Multivariate Logistic Regression Analysis for the Outcome of Acute Pancreatitis Hospitalization for TimePeriods 2002-2005 and 2009-2012 in the Nationwide Inpatient Sample. ${ }^{1}$

\begin{tabular}{|c|c|c|}
\hline Etiologies & Adjusted Odds Ratio (reduced model) $(95 \%$ CI) & Adjusted Odds Ratio (reduced model) $(95 \% \mathrm{CI}$ \\
\hline \multicolumn{3}{|c|}{ Variables without interaction with time-period } \\
\hline Pancreatic Neoplasm & $1.46(1.33-1.60)$ & \\
\hline \multicolumn{3}{|c|}{ Variables having significant interaction with time-period $^{2}$} \\
\hline & $2002-2005$ & $2009-2012$ \\
\hline Gallstone-related & $36.37(35.32-37.46)$ & $29.85(29.09-30.64)$ \\
\hline Chronic pancreatitis & $32.04(30.51-33.64)$ & $35.02(33.94-36.14)$ \\
\hline Alcohol-related & $5.51(5.35-5.67)$ & $4.54(4.44-4.65)$ \\
\hline Hyperlipidemia & $1.58(1.54-1.61)$ & $1.51(1.49-1.53)$ \\
\hline Tobacco use & $1.02(0.99-1.04)$ & $1.06(1.04-1.08)$ \\
\hline Morbid obesity & $0.74(0.71-0.78)$ & $0.81(0.79-0.83)$ \\
\hline Diabetes mellitus & $1.40(1.38-1.43)$ & $1.22(1.21-1.24)$ \\
\hline
\end{tabular}




\section{Table 4}

Multivariate Logistic Regression Analysis for the Outcome Acute Pancreatitis with associated Chronic Pancreatitis: Analysis of the Nationwide Inpatient Sample for the Time-Periods of 2002-2005 and 2009-2012.

\begin{tabular}{|c|c|c|c|}
\hline & Odds Ratio & $(95 \% \mathrm{CI})$ & p-value \\
\hline Time Period & & & $<0.001$ \\
\hline 2002-2005 & Reference & & \\
\hline 2009-2012 & 1.774 & $(1.687-1.865)$ & \\
\hline \multicolumn{4}{|l|}{ Demographics } \\
\hline Age & 0.984 & $(0.983-0.985)$ & $<0.001$ \\
\hline Sex & & & $<0.001$ \\
\hline Male & 1.113 & $(1.082-1.145)$ & \\
\hline Female & Reference & & \\
\hline Race & & & $<0.001$ \\
\hline White & Reference & & \\
\hline Black & 1.188 & $(1.140-1.238)$ & \\
\hline Hispanic & 0.609 & $(0.574-0.647)$ & \\
\hline Other & 0.769 & $(0.701-0.844)$ & \\
\hline Income $^{1}$ & & & $<0.001$ \\
\hline Low & 1.150 & $(1.089-1.215)$ & \\
\hline Moderate & 1.129 & $(1.072-1.188)$ & \\
\hline High & 1.051 & $(1.002-1.102)$ & \\
\hline Very High & Reference & & \\
\hline Insurance & & & $<0.001$ \\
\hline Medicare & 1.500 & $(1.437-1.566)$ & \\
\hline Medicaid & 1.652 & $(1.583-1.725)$ & \\
\hline Private & Reference & & \\
\hline Other & 1.050 & $(1.005-1.097)$ & \\
\hline \multicolumn{4}{|l|}{ Hospital factors } \\
\hline Hospital Type & & & $<0.001$ \\
\hline Rural & 0.639 & $(0.594-0.688)$ & \\
\hline Urban Non-Teaching & 0.709 & $(0.674-0.747)$ & \\
\hline Urban Teaching & Reference & & \\
\hline Hospital Size ${ }^{2}$ & & & $<0.001$ \\
\hline Small & 0.805 & $(0.755-0.858)$ & \\
\hline Medium & 0.948 & $(0.901-0.998)$ & \\
\hline Large & Reference & & \\
\hline Hospital Region & & & $<0.001$ \\
\hline Northeast & Reference & & \\
\hline Midwest & 0.995 & $(0.920-1.077)$ & \\
\hline South & 1.057 & $(0.988-1.131)$ & \\
\hline
\end{tabular}




\begin{tabular}{lrcr} 
& Odds Ratio & $(\mathbf{9 5 \%}$ CI $)$ & p-value \\
\hline West & 0.857 & $(0.795-0.923)$ & \\
\hline Etiologies and Associated Diagnoses & & \\
\hline Gallstone related & 0.186 & $(0.176-0.196)$ & $<0.001$ \\
Alcohol related & 1.332 & $(1.289-1.376)$ & $<0.001$ \\
Hyperlipidemia & 0.870 & $(0.842-0.899)$ & $<0.001$ \\
Pancreatic anomalies & 4.822 & $(4.114-5.652)$ & $<0.001$ \\
Pancreatic neoplasm & 1.020 & $(0.807-1.290)$ & 0.868 \\
Tobacco use & 1.425 & $(1.385-1.467)$ & $<0.001$ \\
Metabolic syndrome & 1.045 & $(0.945-1.156)$ & 0.390 \\
Morbid obesity & 0.606 & $(0.560-0.656)$ & $<0.001$ \\
NAFLD & 0.856 & $(0.803-0.912)$ & $<0.001$ \\
Cholangitis & 0.907 & $(0.771-1.068)$ & 0.242 \\
Acute cholecystitis & 0.342 & $(0.260-0.450)$ & $<0.001$ \\
\hline
\end{tabular}

${ }^{1}$ Estimates of national quartiles are subject to variations on an annual basis: Low $(\$ 1-\$ 38,999)$, Moderate $(\$ 39,000-\$ 47,999)$, High $(\$ 48,000$ $62,999)$, Very high $(\$ 63,000$ or more).

$2 \mathrm{Be}$

Bed size categories are based on hospital beds, and are specific to the hospital's location and teaching status. Bed size assesses the number of short-term acute beds in a hospital.

NAFLD: Non-alcoholic fatty liver disease; CI Confidence Interval. 